\title{
Patient Flow Simulation Using Historically Informed Synthetic Data
}

\author{
Ezra KENNY a , Hamed HASSANZADEH ${ }^{\text {a }}$, Sankalp KHANNA a, \\ Justin BOYLE ${ }^{\text {a }}$ and Sandra LOUISE ${ }^{\text {a }}$ \\ a Australian e-Health Research Centre, CSIRO
}

\begin{abstract}
Hospital overcrowding is a major problem for healthcare systems around the globe. In order to better estimate future demands and adequate resources for coping with such demands, statistical and computerised modelling can be applied. This can then allow healthcare administrators and decision makers to quantify the impacts of various "what-if" scenarios on hospital performance measures. This paper investigates the application of Discrete Event Simulation towards optimising Emergency Department resources while measuring overall length of stay and queuing time of emergency patients as a target performance measure. In particular, we explore strategies for generating historically informed synthetic data that helps the simulation model track patient flow through the target hospital over a future time frame. Using the developed simulation model, several resource configurations are tested using data from one of the busiest emergency departments in the state of Queensland as the baseline while quantifying the impacts of such changes on key patient flow metrics. It was found that adding a single bed (and associated resources) to the emergency department would result in a $23 \%$ decrease in average patient treatment delay.
\end{abstract}

Keywords. patient flow, Discrete Event Simulation, bed optimisation

\section{Introduction}

Hospitals in many countries are increasingly overburdened, due to the ageing population, increased community expectations, and a higher availability of therapeutic interventions. This causes issues with overcrowding and long patient wait times. Increasing the size of existing hospitals is one solution that can help to enhance patient flow. However, adding extra beds to hospitals in the absence of careful examination of current flow blockages within a hospital may result in additional costs to a health care system without improving accessibility to these facilities [1]. Even after pinpointing the bottlenecks in patient flow, it is hard for administrators to quantify the advantage of increasing the capacity and the impact of such expansion on overall flow metrics.

This paper focuses on applying simulation modelling to analyse the effect of capacity variations on patient flow metrics. More specifically, Discrete Event Simulation (DES) was adopted to model patient flow through a hospital's Emergency Department (ED). Different patient flow metrics, such as patient waiting time and average length of stay (LOS), were calculated to quantify the effects of changes in capacity. A synthetic data generator was also developed that learns the trends in the patient flow of the target hospital using a set of historical observations and can then project such demands over a longer future time frame. This simulation model enables health care administrators to 
make more informed decisions by having a better understanding of the effect of different "what-if" scenarios through retrospective and foresight analysis.

\section{Related Work}

There are many examples of simulation applied to operations research in healthcare, to readily and cheaply assess operational scenarios. For example, in a study of an Australian hospital, Khanna et al. assessed the impact of various time-based targets for when patients should be discharged from inpatient wards. They found that discharging $80 \%$ of the day's patient output by 11 a.m. resulted in a $16 \%$ improvement in National Emergency Access Target compliance [2]. Simulation has also been used to analyse patient pathways and determine the most efficient paths available, as in Kovalchuk et al. [3]. A study by Cildoz et al. used simulation and optimisation principles to find ways of developing priority queues (based on patient urgency), while limiting overcrowding [4]. Olabisi and Nwonye used DES in a hospital context, and, in a similar method to this paper, compared actual outcomes (such as average waiting time, queue length) to those from the simulation to calibrate and validate the designed system [5]. Another DESbased approach by Lal et al. found an optimised clinician schedule by aiming to match treatment capacity to patient load over a typical week [6].

A simulation-based study by Best et al. showed promising results by matching staffing to the patient arrival profile, though this simulation used a relatively small sample size [7]. Ahmed and Alkhamis designed a DES system that allows for stochastically varying constraints focusing on emergency department staffing required to maximise patient throughput and minimise waiting time [8]. As a final example, Steward et al. implemented a simulation to investigate workflow associated with patient care streams [9]. In this scenario, patient journey was determined by their care stream (critical, diagnostic, therapeutic, fast-track, or resuscitation), and the authors found this method reduced both patient LOS and wait time. While there are many DES studies applied to the healthcare field, none report on the use of synthetic data modelled from historical data, which this paper explores. Such synthetic data may allow exploration of system impacts beyond the constraints imposed by observed data. This includes exploring a longer time period or assessing resources such as wards or staffing that are not historically included in the data.

\section{Methodology}

\subsection{Discrete Event Simulation}

In large-scale healthcare scenarios, it can be difficult, risky and expensive to experiment with protocols, operational scenarios, and resources. This often means that simulation modelling is among the safest and least costly ways of analysing these situations. One of the widely applied techniques to model systems that include wait times and queueing is DES. Within DES, resources are objects or services that can be used (e.g. hospital wards) with a finite capacity (e.g. number of beds), and the objects of interest (e.g. patients) will 


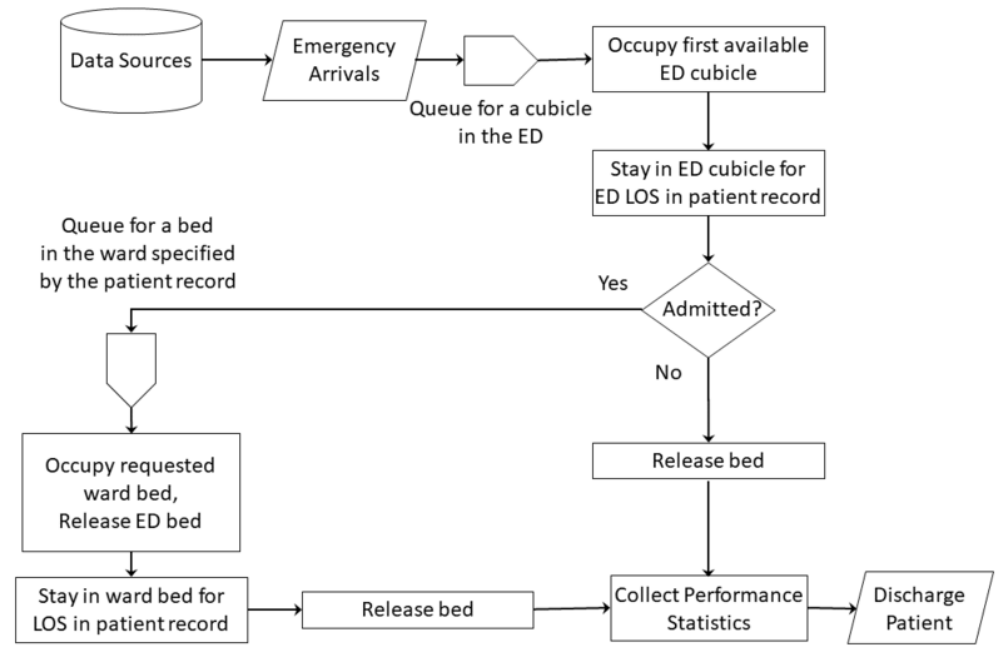

Figure 1. ED patient flow modelling.

queue for desired resources, hold them for a time, and then release them in interactions called "events".

Figure 1 shows the flowchart that was used to create the DES simulation. It involves sorting through the data source to obtain emergency arrivals, which are entered into a queue for an ED bed (this is treatment delay). Once one is available, the patient occupies that bed for the LOS specified in their patient record. Then, if they are admitted to hospital, they queue for the ward they are to be admitted to (this is ED discharge delay). They occupy a bed in that ward for the LOS in their patient record, and then release the bed before performance statistics are collected and the patient is discharged. If the patient is not admitted, after their ED LOS is complete they release the bed, performance statistics are collected and the patient is discharged. This simulation model enables the quantification of the impacts of changing capacities in both ED and inpatient wards (in terms of number of beds/resources), but analysis reported in this paper will focus on the effect a change in ED beds will have on treatment delay.

By giving finite capacities to the resources (i.e. ED beds) in the simulation, queues begin to form once all the beds are occupied. The time that patients wait in the queue to obtain a bed is calculated as their waiting time and materialised as treatment or discharge delays. The priority of patients for receiving treatment services was also modelled in this simulation by considering their urgency level, which was assessed during the triage process.

\subsection{Synthetic Data Generation Using Historical Observations}

The above-mentioned simulation model can be developed to mimic the historic observations and to quantify the impacts of various changes through a retrospective analysis. In order to enable the model to simulate such impacts in a longer future time frame that exceeds the historic observations, a set of synthetically-generated data should be used. However, the quality of the generated data directly affects the accuracy of the quantified effects returned by the model. 
The method of Kernel Density Estimation was used to generate new data. Kernel Density Estimation uses all of the target hospital data, centring a kernel function at each point. Since it keeps the distributions (in arrival times, urgency category, etc.) inherent in the historical information, the synthetic data can accurately reflect the patient flow in the target hospital.

A number of kernels were investigated for this simulation, including Gaussian, top hat, linear and cosine kernels. The Gaussian kernel most closely fit the actual distributions and so it was adopted in our synthetic data generator. The generator was then used to produce patient arrivals on a daily basis. Apart from the admission rates, the generator reproduced several patient characteristics including their Australasian Triage Scale urgency category, discharge destination (whether they were subsequently admitted or discharged home after ED treatment), inpatient admitted wards, and the LOS in each ward. With the actual data as an input, the generator learns the distribution of such characteristics and any given amount of data could be output.

This analysis focused on a Queensland metropolitan hospital with one of the busiest emergency departments in the state, seeing more than 88,000 presentations each year. A de-identified data extract for a period of 18 months (from 1 July 2018 to 31 December 2019) of all ED admissions to the target hospital was used to train the synthetic data generator. Ethics approval was obtained from the Hospital's Human Research Ethics Committee. The data was cleaned to remove any inconsistent patient records, and missing values were replaced with adjacent timestamps where applicable. Patients who died within the ED or who did not stay for treatment contributed to a small fraction of the total patients, and were removed from the data. The details for each patient contained timestamps showing their journey throughout the hospital including time they arrived at ED, were triaged, treated, were ready for discharge, departed from ED and where applicable, subsequent inpatient admission and discharge times.

In order to validate the data generator, a set of synthetic data for 18 months was generated. The output performance measures of the simulation model using actual and synthetic data sets were compared to ensure that the synthetic data reflected actual patterns. Following this, an extended set of synthetic data spanning 30 months was generated, which was then used to report the performance measures of the simulated hospital environment for an additional successive year.

\subsection{Experimental Setup}

Within the Python programming language, a module called Scikit-learn was used to investigate and adopt a suitable kernel density function [10]. The simulation was developed using SimPy, which is a library in Python specifically designed for DES. The synthetic data was input to the program, which simulated the movement of patients through the ED, and calculated the simulated time taken for triage, waiting for a bed and treatment. The modelling of the priority of patients to receive treatment service was performed using patients' urgency category as the priority value in the SimPy's "Priority Resource".

A number of ED-related performance measures were calculated to quantify the impacts of changing ED bed capacity. These include average ED LOS, and average waiting time before and after treatment (treatment and discharge delay, respectively). The results were also further separated by admitted, non-admitted, and all patient populations. 
The simulation model was run with a configuration that meant the ED waiting room has infinite capacity, and the ED capacity reflects the availability of both cubicles/beds and staff. In the absence of a pre-defined ED capacity, the model was calibrated with an ED capacity that resulted in measures closest to the actual outputs.

\section{Results and Discussion}

Several bed configurations were investigated using the developed simulation model. Table 1 shows results for just one of the ED performance measures assessed: the percentage decrease in treatment delay when increasing the number of ED beds, compared to the baseline (i.e. with the calibrated ED capacity). The results are given for three patient cohorts: admitted, non-admitted, and all patients. The simulated results in Table 1 show two different settings: 1) using 18 months of data, and 2) using 30 months of data. In the former setting, the first 6 months was considered as the warm-up period while the first 18 months was the warm-up period for the latter setting. In both settings, the results were calculated on the final 12 months of the data.

It can be seen that the reduction in average treatment delays were similar for admitted, non-admitted, and all patients as the number of ED beds increased. There was approximately a $23 \%$ reduction in treatment delays as a result of adding just one bed. It can be observed that the percentage decrease was correlated to the number of additional beds and a similar pattern in average percentage decrease was exhibited when using the two lengths of data. This reduction can have a significant impact on total ED LOS, especially for non-admitted patients, whose longest waiting time on average was ED treatment delay.

Looking at the two sets of data, it can be observed that the 30 months setting displayed slightly less delay reduction than the 18 month setting, especially on non-admitted patients. This can be due to the increase in the hospitalisation rate in a longer future time frame. The reason that non-admitted patients experienced greater treatment delays during the 30 months of simulation than the admitted patients is due to their lower average urgency priorities, and hence their longer waiting times in the queue for receiving treatment services.

As shown in Table 1, it became apparent that significant changes to waiting times can be achieved by adding a small number of beds. However, it should be noted that adding a single bed is associated with purchasing further medical equipment and employing more staff, which has a considerable associated cost. Although adding 4 beds to the ED may not be feasible, from a management perspective, it is still worthwhile tracking where resources would have the biggest impact on patient flow metrics and when the addition of resources ceases to have a large enough impact on patient throughput.

Table 1. Average percentage decrease in treatment delay with increase in ED beds.

\begin{tabular}{ccccccc}
\hline $\begin{array}{c}\text { Added } \\
\text { ED } \\
\text { bed(s) }\end{array}$ & \multicolumn{2}{c}{ Percentage decrease (\%), 18 months } & \multicolumn{3}{c}{ Percentage decrease (\%), 30 months } \\
\cline { 2 - 7 } & All & Admitted & $\begin{array}{c}\text { Non- } \\
\text { Admitted }\end{array}$ & All & Admitted & $\begin{array}{c}\text { Non- } \\
\text { Admitted }\end{array}$ \\
\hline 1 & 23.21 & 23.10 & 23.24 & 22.71 & 23.21 & 22.79 \\
2 & 41.48 & 41.55 & 41.46 & 39.93 & 41.39 & 39.89 \\
3 & 55.54 & 55.03 & 55.70 & 53.72 & 55.84 & 53.60 \\
4 & 66.86 & 66.17 & 67.08 & 64.60 & 66.96 & 64.52 \\
\hline
\end{tabular}




\section{Conclusion}

By analysing data from one of Queensland's busiest hospitals, we were able to find trends and bottlenecks within patients' journeys. A synthetic data generator based on historical observations was developed that can provide de-identified data over a customised future time frame. A simulation was built that models patient movement throughout the ED, allowing for the analysis of "what-if" scenarios. The results of these scenarios showed that with 1 added ED bed, average treatment delay times were decreased by $23 \%$. This rose to $41 \%$ when 2 extra beds were added, and further reductions in delay were observed as ED capacity increased.

While this simulation has some limitations, in that it does not individually model staff or waiting room capacities, it produces insightful results for the hospital management team to make more informed decisions. There is also broad scope for further research, including analysis of ED discharge delays, and inpatient journeys throughout the hospital. This analysis could include clustering patients by their pathway, clustering wards with similar functionality to reduce queuing and considering utilisation of resources $-85 \%$ occupancy is often suggested as optimal [11]. It could also be useful to find and recommend elimination of inefficient patient pathways, and include specific analysis of elective patients and ambulance arrivals.

\section{References}

[1] Morley C, Unwin M, Peterson GM, Stankovich J, Kinsma L. Emergency department crowding: A systematic review of causes, consequences and solutions. PLoS One. 2018;13(8):e0203316. doi: 10.1371/journal.pone.0203316.

[2] Khanna S, Sier D, Boyle J, and Zeitz K. Discharge timeliness and its impact on hospital crowding and emergency department flow performance. Emergency Medicine Australasia, 2016;28:164-170. doi: $10.1111 / 1742-6723.12543$.

[3] Kovalchuk SV, Funkner AA, Metsker OG, Yakovlev AN. Simulation of patient flow in multiple healthcare units using process and data mining techniques for model identification. Journal of Biomedical Informatics. 2018;82:128-142. doi: 10.1016/j.jbi.2018.05.004.

[4] Cildoz M, Mallor F, Ibarra A. Analysing the ED patient flow management problem by using accumulating priority queues and simulation-based optimization. Proceedings of the 2018 Winter Simulation Conference (WSC). Gothenburg, Sweden. p. 2107-2118, doi: 10.1109/WSC.2018.8632323.

[5] Olabisi U, Nwonye C. Discrete-event Simulation Modeling of Patient Flow in Healthcare systems. International Journal of Advancement in Physical Sciences. 2012;4(1):69-86.

[6] Lal TM, Roh T, Huschka T. Simulation Based Optimization: Applications in Healthcare. Proceedings of the 2015 Winter Simulation Conference. Huntington Beach California, USA. doi: 10.1109/WSC.2015.7408251.

[7] Best AM, Dixon CA, Kelton WD, Lindsell CJ, Ward MJ. Using discrete event computer simulation to improve patient flow in a Ghanaian acute care hospital. Am J Emerg Med. 2014;32(8):917-922. doi:10.1016/j.ajem.2014.05.012.

[8] Ahmed MA, Alkhamis TM. Simulation optimization for an emergency department health-care unit in Kuwait. European Journal of Operational Research. 2009;198(3):936-942. doi:10.1016/j.ejor.2008.10.025.

[9] Steward D, Glass TF, Ferrand YB. Simulation-Based Design of ED Operations with Care Streams to Optimize Care Delivery and Reduce Length of Stay in the Emergency Department. J Med Syst. 2017;41(162). doi:10.1007/s10916-017-0804-6.

[10] Pedregosa F, Varoquaux G, Gramfort A, Michel V, Thirion B, Grisel O, et al. Scikit-learn: Machine learning in Python. Journal of Machine Learning Research, 2011;12:2825-2830.

[11] Bagust A, Place M, Posnett JW. Dynamics of bed use in accommodating emergency admissions: stochastic simulation model. Br. Med. J. 1999;319(7203):155-158. doi:10.1136/bmj.319.7203.155. 